\title{
Performance Comparison between FBMC and OFDM in MIMO Systems under Channel Uncertainty
}

\author{
Miquel Payaró*, Antonio Pascual-Iserte*†, and Montse Nájar*† \\ ${ }^{*}$ Centre Tecnològic de Telecomunicacions de Catalunya \\ Castelldefels, Barcelona, Spain \\ ${ }^{\dagger}$ Department of Signal Theory and Communications \\ Universitat Politècnica de Catalunya (UPC), Barcelona, Spain \\ Emails: miquel.payaro@cttc.es, antonio.pascual@upc.edu, montse.najar@cttc.es
}

\begin{abstract}
In this paper we present a performance comparison between two different multi-carrier transmission techniques: OFDM, based on the FFT and cyclic prefix (CP) addition; and FBMC (FilterBank MultiCarrier), which, as indicated by its name, is based on a filterbank architecture. For both schemes, we propose a joint beamforming design to be applied in multi-inputmulti-output (MIMO) systems, which requires channel state information (CSI) at both communication ends.

If perfect CSI is assumed, FBMC presents a higher energyefficiency since it does not require a $\mathrm{CP}$, differently to OFDM. However, in the imperfect CSI case, the opposite occurs: while in OFDM the presence of errors in the CSI does not cause intersymbol interference (ISI) and inter-carrier interference (ICI), in the FBMC case we show, both analytically and through simulation results, that imperfections in the CSI imply both ISI and ICI, which leads to an energy-efficiency loss. To mitigate this loss, we propose a novel robust receive beamforming strategy. In the simulations section, the performance of FBMC with robust beamforming is shown to outperform OFDM even in imperfect CSI conditions.

The characterization of the tradeoff between energy-efficiency and robustness against channel uncertainty, both with robust and non-robust strategies, is, therefore, the objective of the present paper and also the motivation for future robust designs for FBMC multiantenna systems.
\end{abstract}

\section{INTRODUCTION}

Multi-carrier (MC) modulations have become the key physical layer transmission technology adopted in not only current broadband communication systems, such as digital subscriber line (DSL), wireless local area network (WLAN), worldwide interoperability for microwave access (WiMAX), or long term evolution (LTE); but also in digital video and audio broadcasting (DVB-T, T-DAB) [1], [2]. The basic idea behind MC modulation is the division of the digital bit stream to be transmitted through a wideband channel into a set of parallel bit streams with a lower rate. Each of these bit-streams

This work was partially supported by the Catalan Government under grants 2009 SGR 1046 and 2009 SGR 891, by the Spanish Government under project TEC2008-06327-C03 (MULTI-ADAPTIVE), and by the European Commission under projects ICT-FP7-211887 (PHYDYAS) and ICT-FP7216715 (NEWCOM++). requires a lower bandwidth and is transmitted using a different frequency subband of the whole channel in such a way that the channel can be approximated as frequency flat for each bitstream. The main benefit of this conversion of the wideband channel into a set of parallel narrowband flat fading channels is that this substantially reduces the complexity of the receiver in terms of equalization and also channel estimation.

There exist several practical implementations of the MC approach. The most widely known MC modulation is orthogonal frequency division multiplexing (OFDM) [2]. In this scheme, each symbol in each parallel stream is modulated by means of a carrier with a rectangular window, where the carriers corresponding to different streams have a different frequency. The simplicity of the scheme relies on the orthogonality among such carriers which, in turn, requires the addition of a so-called cyclic prefix (CP). The function of this $\mathrm{CP}$, which consists in the enlargement of the symbol duration, is to assure the orthogonality even with a non flat fading channel. The main disadvantage is that the use of the $\mathrm{CP}$ implies a reduction of the energy efficiency of the transmission.

An alternative to OFDM which does not require the use of a $\mathrm{CP}$ is the filterbank multicarrier (FBMC) modulation, which is also known as OFDM/OQAM [3], [4]. The main difference of FBMC with respect to OFDM is that instead of rectangular windows, a more advanced basic pulse, also called prototype filter, is used, which can reduce the out-of-band frequency leakage and help to fulfill more stringent spectral masks. The use of FBMC with appropriate filter prototypes allows to obtain an almost negligible inter-symbol interference (ISI) and inter-carrier interference (ICI) assuming that the channel coherence bandwidth is high enough.

In addition to MC technologies, also many systems have adopted spatial diversity based on the use of multiple antennas at both the transmitter and the receiver as an alternative to boost system capacity [5]. In this sense, multi-input-multioutput (MIMO) channels have become a key research focus in last years. In this paper we propose a scheme to jointly exploit MC modulations and MIMO systems by means of 
a joint beamforming approach per carrier [6]. This joint beamforming solution requires having a channel estimate, also called channel state information (CSI), at both ends of the communication system. In practical systems, this CSI will not be perfect due to several reasons, such as the presence of estimation noise, quantization errors, etc. [7]. In this paper we will analyze how the imperfections in the CSI affect the performance of MIMO-OFDM and MIMO-FBMC under joint beamforming. It will be shown that in the FBMC case, an important ISI and ICI component may appear additionally. To cope with this undesired effect, we propose a novel robust receive beamformer design, which is adapted to the particularities of FBMC signals.

The rest of this paper is organized as follows. In Section II we deal with the system model for FBMC in singleinput-single-output (SISO) channels. Next, in Section III we introduce the MIMO system and study the effects of imperfect CSI. The robust design proposed to cope with the imperfect CSI is studied in Section IV. The simulation results depicting the performance of the studied schemes can be found in Section V and, finally, the conclusions are drawn.

\section{THE FBMC ARCHITECTURE}

While the system model for OFDM systems is well known in the communications community [2], less is known about FBMC. In the following subsections, we introduce the basic concepts and the signal model behind FBMC for SISO channels.

\section{A. Initial basic considerations}

The transmitted signal in FBMC is the sum of the outputs of a bank of $K$ filters, $f_{k}$, whose length is given by $L$. Thus, at any given time instant $m$, the discrete-time baseband equivalent of the transmitted signal, $x(m)$, is given by

$$
x(m)=\sum_{n=-\infty}^{\infty} \sum_{k=0}^{K-1} d_{k, n} f_{k}(m-\tau n),
$$

where $\left\{d_{k, n}\right\}$ denotes the input symbol sequence of the $k$-th filter and $\tau$ is the symbol period. Observe that the index $n$ is, similarly to $m$, a time index. However, whereas $m$ indicates the time in the filter-sample and transmitted signal domains, $n$ corresponds to the symbol index. For the time being, we will not bother about how the filterbank input symbols $d_{k, n}$ are obtained as a function of the data symbols.

Once the signal has been transmitted, it undergoes a transformation which is determined by the channel impulse response $h(m)$ and the noise $w(m)$ obtaining, at the receiver side, a signal $y(m)$ which is given by

$$
y(m)=h(m) * x(m)+w(m),
$$

where $*$ denotes the convolution operation. The received signal $y(m)$ is then processed by a bank of $K$ filters $g_{k}$ (which are the matched filters to $f_{k}$ ) to obtain the output $r_{k, n}$ of this communications system:

$$
r_{k, n}=\left.g_{k}(m) * y(m)\right|_{m=m_{0}(n)},
$$

where $m_{0}(n)=\tau n+\tau_{0}$, which corresponds to the decision time at the receiver side and where $\tau_{0}$ is the time index that maximizes $\left|f_{k}(m) * g_{k}(m)\right|$ plus, possibly, the extra delay introduced by the channel.

Provided that the coherence bandwidth of the channel is high enough, the input-output response of the filterbank system in (3), particularized at symbol-time $n_{0}$ and frequency $k_{0}$ can be well approximated by [8]

$$
r_{k_{0}, n_{0}} \cong \sum_{n=-\infty}^{\infty} \sum_{k=0}^{K-1} H_{k} t_{k_{0}-k, n_{0}-n} d_{k, n}+w_{k_{0}, n_{0}},
$$

where $H_{k}$ is the $k$-th element of the $K$-point Fourier transform of the impulse response $h(m), w_{k_{0}, n_{0}}$ represents the noise sample, and $t_{k_{0}-k, n_{0}-n}$ is given by

$$
t_{k_{0}-k, n_{0}-n}=\left.f_{k_{0}}(m) * g_{k}\left(m+\tau n_{0}\right)\right|_{m=m_{0}(n)}
$$

and represents the transmultiplexer response, i.e., the ISI and ICI "footprint" centered at $k=k_{0}$ and $n=n_{0}$ obtained when a single symbol $d_{k_{0}, n_{0}}=1$ is transmitted.

\section{$B$. Filter parameters design and the OQAM modulation}

The performance of the architecture described above depends mainly on the actual response of the filters $f_{k}$, which characterizes their frequency shape. There exist many references in the literature which deal with filter design (see [1] and references therein), however we will stick to the proposal by Bellanger in [9], because it presents an excellent trade-off between time and frequency localization. In addition, the filters designed in [9] have the advantage that they are uniform, i.e., all the filters can be obtained as a frequency-shifted version of a prototype $f_{0}$ :

$$
f_{k}(m)=f_{0}(m) \exp \left(\mathrm{j} \frac{2 \pi k m}{K}\right),
$$

where $1 / K$ is the frequency separation between subbands (we recall that $K$ is the total number of carriers).

For example, we can take the design in [9] with filter length $L=2048$, symbol period $\tau=256$, and $K=512$ carriers. $^{1}$ For this specific choice of the filter design parameters, the transmultiplexer relation $t_{k_{0}-k, n_{0}-n}$, as given in (5), is depicted in Fig. 1. At first sight, this transmultiplexer response does not appear to be well suited for communication purposes, since the amount of ISI and ICI is remarkable.

Now, enter the OQAM modulation, which is the modulation used in FBMC systems. The main feature of this modulation is that the symbols $d_{k, n}$ are obtained, as a function of the data information symbols, according to

$$
d_{k, n}=\mathrm{j}^{(k+n)} s_{k, n} \equiv \theta_{k, n} s_{k, n},
$$

where $s_{k, n}$ are real PAM symbols. The effect of the $\theta_{k, n}$ factor is to create a time-frequency pattern of alternate real and

\footnotetext{
${ }^{1}$ Observe that the symbol period $\tau$ is half the inverse of the frequency separation between subbands, $K$. The choice of this unusual relation, which is a feature in FBMC systems and seems to contradict the Nyquist criterion, will become clear in the following.
} 


\begin{tabular}{|c|c|c|c|c|c|c|c|c|c|}
\hline & -4 & -3 & -2 & -1 & 0 & 1 & 2 & 3 & 4 \\
\hline-2 & 0 & 0.0006 & -0.0001 & 0 & 0 & 0 & -0.0001 & 0.0006 & 0 \\
\hline-1 & 0.0054 & $0.0429 \mathrm{j}$ & -0.1250 & $-0.2058 j$ & 0.2393 & $0.2058 \mathrm{j}$ & -0.1250 & $-0.0429 j$ & 0.0054 \\
\hline 0 & 0 & -0.0668 & 0.0002 & 0.5644 & 1 & 0.5644 & 0.0002 & -0.0668 & 0 \\
\hline 1 & 0.0054 & $-0.0429 j$ & -0.1250 & $0.2058 \mathrm{j}$ & 0.2393 & $-0.2058 j$ & -0.1250 & $0.0429 j$ & 0.0054 \\
\hline 2 & 0 & 0.0006 & -0.0001 & 0 & 0 & 0 & -0.0001 & 0.0006 & 0 \\
\hline
\end{tabular}

Fig. 1. Graphical representation of the time-frequency response of the FBMC system considered in this work. Thanks to the OQAM modulation used in FBMC systems the effective time-frequency response will be reduced to only the shaded elements in the table. This implies that the ISI and ICI terms are, in the perfect CSI case, essentially negligible.

imaginary symbols $d_{k, n}$, i.e., if $d_{k, n}$ is real, then all $d_{k+1, n}$, $d_{k-1, n}, d_{k, n+1}$, and $d_{k, n-1}$ are pure imaginary and viceversa.

Then, at the receiver side where complexity is to be kept at a minimum, the signal $r_{k_{0}, n_{0}}$ given in (3) and (4) is multiplied by $\theta_{k_{0}, n_{0}}^{*}$ and divided by $H_{k_{0}}$ to equalize the channel, obtaining

$$
\begin{aligned}
\frac{\theta_{k_{0}, n_{0}}^{*} r_{k_{0}, n_{0}}}{H_{k_{0}}}= & s_{k_{0}, n_{0}}+\sum_{(k, n) \in \mathcal{R}} \frac{H_{k_{0}-k}}{H_{k_{0}}} t_{k, n}\left( \pm s_{k_{0}-k, n_{0}-n}\right) \\
& +\mathrm{j} \sum_{(k, n) \in \mathcal{I}} \frac{H_{k_{0}-k}}{H_{k_{0}}} t_{k, n}\left( \pm s_{k_{0}-k, n_{0}-n}\right) \\
\cong & s_{k_{0}, n_{0}}+\sum_{(k, n) \in \mathcal{R}} t_{k, n}\left( \pm s_{k_{0}-k, n_{0}-n}\right) \\
& +\mathrm{j} \sum_{(k, n) \in \mathcal{I}} t_{k, n}\left( \pm s_{k_{0}-k, n_{0}-n}\right)
\end{aligned}
$$

where

$$
\begin{gathered}
\mathcal{R}=\{(2,4),(2,2),(2,0),(2,-2),(2,-4),(0,4),(0,2), \\
(0,-2),(0,-4),(-2,4),(-2,2),(-2,-2),(-2,-4)\},
\end{gathered}
$$

the set $\mathcal{I}$ contains the remaining elements in the full set $k \in$ $[-2,2], n \in[-4,4]$, the high coherence bandwidth assumption has been used to obtain (9), and where, with a slight abuse of notation, the sign \pm has been used to indicate the sign of $\theta_{k_{0}, n_{0}}^{*} \theta_{k_{0}-k, n_{0}-n}{ }^{2}$

Finally, to recover the real-valued PAM symbols we take the real part in (9), which also implies that the pure-imaginary ISI

\footnotetext{
${ }^{2}$ Observe that in case that the channels in adjacent subcarriers are not exactly equal, then the factor $H_{k_{0}-k} / H_{k_{0}}$ will not be exactly equal to 1 (and its phase will be close to but not exactly 0 ) and this will imply that, after taking the real part, some additional ISI and ICI will appear. Nevertheless, these extra components can be cancelled by a two-dimensional time-frequency equalizer such as those described in [10]. The presence of an equalizer renders this effect out of the scope of the present paper. Consequently we will consider that this extra ISI and ICI is equalized in our system and we will focus exclusively on the effects produced by an imperfect CSI as described in the next section.
}

and ICI elements in $\mathcal{I}$ are eliminated, leaving only the elements in $\mathcal{R}$, which, as it can be observed from Fig. 1, produce an almost negligible ISI and ICI. Observe that the elements in $\mathcal{R}$ correspond the shaded elements in Fig. 1, whose magnitude is almost negligible. Finally, the estimated data symbol is given by

$$
\hat{s}_{k_{0}, n_{0}}=\Re \mathrm{e}\left\{\frac{\theta_{k_{0}, n_{0}}^{*} r_{k_{0}, n_{0}}}{H_{k_{0}}}\right\} .
$$

This effective lack of ISI and ICI implies that the subcarriers in FBMC behave as quasi-orthogonal parallel channels and this is the reason why, in the literature, the FBMC communication scheme is also referred to as OFDM/OQAM.

Finally, a comment on the rate of FBMC systems is in order. Since the information symbols $s_{k, n}$ are forced to be real instead of complex, this would imply a spectrum use inefficiency by a factor of 2 . However, this sub-optimal use of the spectrum is compensated by the choice $\tau=K / 2$ for the symbol period in (1) which is half the inverse of the frequency separation, thus doubling the rate. The net effect is that the rate is the same as in a traditional OFDM system with complex signaling and symbol delay given by $K$.

\section{EXTENSION TO MIMO SYSTEMS AND IMPERFECT CSI}

\section{A. Model for the multiantenna case}

In this section, we address the possibility of combining the MC modulation (either OFDM or FBMC) with the use of $n_{T}$ antennas at the transmitter and $n_{R}$ antennas at the receiver, configuring a MIMO channel. Thanks to this spatial diversity, the system performance and capacity can be boosted. There are several ways to exploit such spatial diversity depending basically on the quality and quantity of the CSI available during the design in the transmitter and/or the receiver and the allowed complexity.

In this work, we take the approach of single joint beamforming per carrier, which requires full knowledge of the MIMO channel estimate at both sides of the communication system and consists in transmitting a single symbol stream 


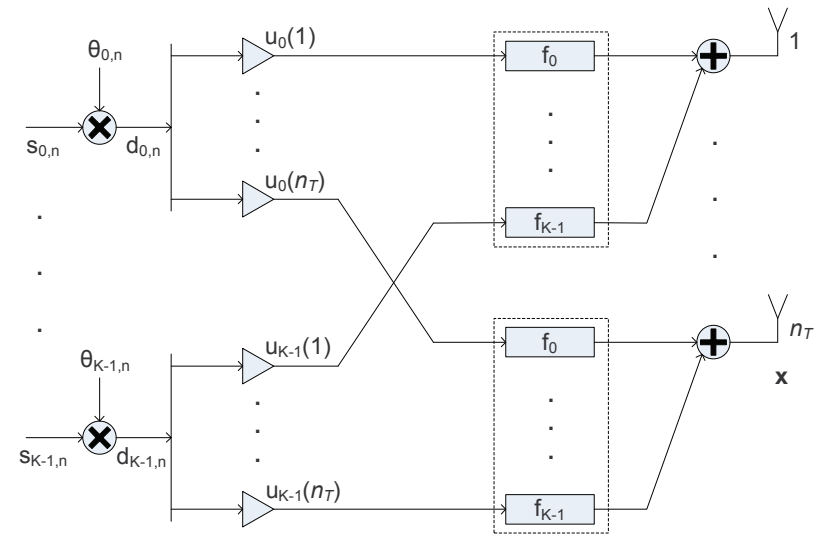

Fig. 2. Transmitter architecture for the MIMO-FBMC system studied in this paper. Observe that the signal transmitted by each antenna is the superposition of the outputs of a filterbank (boxed in a dashed line). For MIMO-OFDM the structure is analogous, but replacing the filterbanks by a concatenation of an IFFT block and a CP addition block.

per carrier (see Figs. 2 and 3 for a block diagram of the transceiver structure). This single stream at each carrier, $d_{k, n}$, is multiplied at the transmitter by a set of weights (one per antenna) collected in the so called transmit beamvector $\mathbf{u}_{k} \in \mathbb{C}^{n_{T}}$. Then, each component of $\mathbf{u}_{k} d_{k, n}$ is independently filtered by its corresponding filter $f_{k}$ before being sent through the transmit antennas, obtaining for $\mathrm{FBMC}^{3}$ :

$$
\mathbf{x}(m)=\sum_{n=-\infty}^{\infty} \sum_{k=0}^{K-1} f_{k}(m-\tau n) \mathbf{u}_{k} d_{k, n},
$$

where, in the MIMO case, the $j$-th entry of vector $\mathbf{x}(m)$ represents the signal transmitted through the $j$-th antenna.

The MIMO channel is now expressed through a matrix $\mathbf{h}(m) \in \mathbb{C}^{n_{R} \times n_{T}}$, whose $(i, j)$-th entry contains the channel impulse response between $j$-th transmit and $i$-th receive antennas. The received vector $\mathbf{y}(m) \in \mathbb{C}^{n_{R}}$ is simply the matrix convolution between the channel and the transmitted vector plus the noise vector, $\mathbf{y}(m)=\mathbf{h}(m) * \mathbf{x}(m)+\mathbf{w}(m)$.

At the receiver, the incoming vector $\mathbf{y}(m)$ is fed into the receive filterbanks (one per antenna) obtaining, at each frequency,

$$
\mathbf{z}_{k, n}=\left.g_{k}(m) * \mathbf{y}(m)\right|_{m=m_{0}(n)},
$$

which can be seen as the received vector for each frequency carrier and where $m_{0}(n)$ is the same as in Section II. Next, each one of these vectors $\mathbf{z}_{k, n}$ is multiplied by its corresponding receive beamvector, which is designated by $\mathbf{v}_{k}$. Thus, we obtain, similarly as in (3) for the SISO case, the signal $r_{k, n}$, which now reads as

$$
r_{k, n}=\mathbf{v}_{k}^{H} \mathbf{r}_{k},
$$

where we have used $(\cdot)^{H}$ to denote the Hermitian operator. Finally, the signal $r_{k, n}$ is equalized to obtain the estimation of $s_{k, n}$ (the equalization details are given later).

\footnotetext{
${ }^{3}$ For the system model in MIMO-OFDM the reader is referred to [6]
}

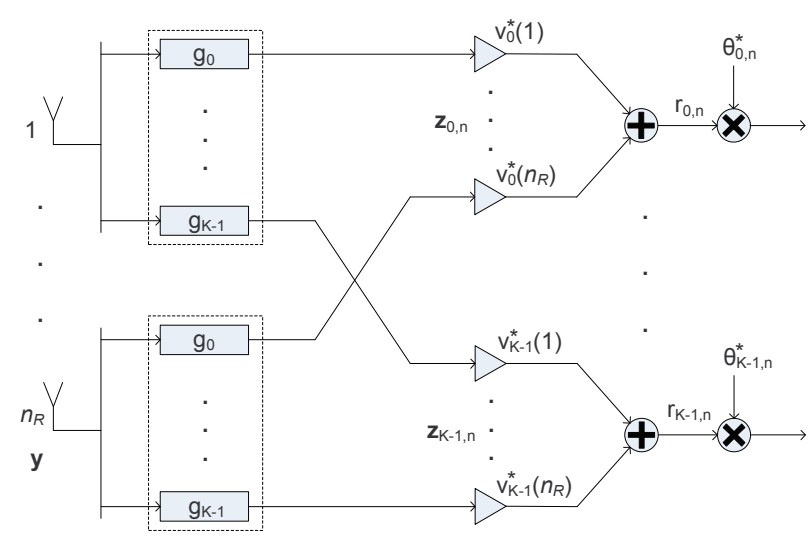

Fig. 3. Receiver architecture for the MIMO-FBMC system studied in this paper. Before detecting the transmitted data, a frequency-time equalizer may be placed at the output of this architecture. For MIMO-OFDM the structure is analogous but the filterbanks are replaced by a CP-removal and FFT operation.

Observe that, in general, the MIMO channel is expected to be frequency selective and, consequently, the transmit and receive beamvectors, $\mathbf{u}_{k}$ and $\mathbf{v}_{k}$, can be different for each frequency as it has been explicitly indicated by the index $k$.

\section{B. Joint beamformer design and equivalent SISO channel}

The design of the beamvectors, $\mathbf{u}_{k}$ and $\mathbf{v}_{k}$, depends on the channel matrix $\mathbf{h}(m)$. As pointed out in Footnote 2, we assume that the ISI and ICI components with perfect CSI are equalized (this is true in OFDM and an approximation in FBMC), therefore, the beamvectors for a specific carrier depend only on the channel response at such subband.

In the case of single joint beamforming, it was shown in [6] that the SNR-maximizing transmit beamvector at the $k$ th carrier is equal to a scaled version of the right singular vector $\varphi_{k}$ associated to the maximum singular value $\lambda_{k}$ of the matrix $\mathbf{H}_{k}$, whose $(i, j)$-th element is the $k$-th component of the $K$-point Fourier transform of the channel between $j$ th transmit and $i$-th receive antennas (given by $[\mathbf{h}(m)]_{i, j}$ as pointed out in the previous section). The scale factor is equal to the square root of the power allocated to such carrier, i.e., $\mathbf{u}_{k}=\sqrt{p_{k}} \boldsymbol{\varphi}_{k}$, such that the sum of allocated powers is equal to the total transmitted power $P_{T}=\sum_{k} p_{k}$. At the receiver, the beamvector $\mathbf{v}_{k}$ is equal to the spatial matched filter

$$
\mathbf{v}_{k}^{H}=\alpha_{k}\left(\mathbf{H}_{k} \boldsymbol{\varphi}_{k}\right)^{H} \equiv \boldsymbol{\psi}_{k}^{H},
$$

where $\alpha_{k}$ is such that the norm $\left\|\boldsymbol{\psi}_{k}\right\|$ is equal to 1 . The resulting equivalent SISO channel gain of the joint beamforming scheme is thus given by

$$
C_{k}=\mathbf{v}_{k}^{H} \mathbf{H}_{k} \mathbf{u}_{k}=\boldsymbol{\psi}_{k}^{H} \mathbf{H}_{k} \sqrt{p_{k}} \boldsymbol{\varphi}_{k}=\lambda_{k} \sqrt{p_{k}} .
$$

Concerning the power allocation, several approaches are possible depending on the adopted figure of merit for the system or optimization criterion, such as the minimization of the probability of error, the minimization of the mean square error, the maximization of a mean value of the SNRs at the different carriers, etc. In [6], all these possibilities are 
studied. In this paper we will only consider two of them as meaningful examples: uniform power allocation (UPA), where $p_{k}=P_{T} / K, \forall k$ and the minimum effective probability of error (MEPE) where

$$
p_{k}=\max \left\{\frac{2 \log \left(\lambda_{k}\right)-\mu}{\lambda_{k}^{2}}, 0\right\},
$$

where we recall that $\lambda_{k}$ is the maximum singular value of $\mathbf{H}_{k}$ and $\mu$ is such that the power constraint is fulfilled.

\section{Imperfect CSI}

In practice, only a channel estimate $\widetilde{\mathbf{H}}_{k}$ is available to perform the design of the transmit and receive beamvectors. Consequently, if we take a naive approach and design the beamvectors without taking into account that the channel is imperfect, the transmit and receive beamvectors in the imperfect CSI case will correspond to scaled versions of the right and left singular vectors of $\widetilde{\mathbf{H}}_{k}$ associated with its maximum singular value, which are denoted by $\mathbf{u}_{k}=\sqrt{\tilde{p}_{k}} \tilde{\varphi}_{k}$ and $\mathbf{v}_{k}=\tilde{\psi}_{k}$, respectively.

This implies that the actual equivalent channel, defined in the imperfect CSI case as

$$
\bar{C}_{k}=\tilde{\boldsymbol{\psi}}_{k}^{H} \mathbf{H}_{k} \sqrt{\tilde{p}_{k}} \tilde{\boldsymbol{\varphi}}_{k},
$$

has a phase and amplitude mismatch with respect to the estimated equivalent channel given by

$$
\begin{aligned}
\tilde{C}_{k}=\tilde{\boldsymbol{\psi}}_{k}^{H} \tilde{\mathbf{H}}_{k} \sqrt{\tilde{p}_{k}} \tilde{\boldsymbol{\varphi}}_{k}=\tilde{\lambda}_{k} \sqrt{\tilde{p}_{k}}, & \\
& \left(\tilde{C}_{k} \in \mathbb{R}^{+} \text {by construction }\right) .
\end{aligned}
$$

While this is not a critical problem in OFDM, it can really be in the case of FBMC as it is shown next.

At the output of the equalizer, the estimate of the data symbol $\hat{s}_{k_{0}, n_{0}}$ is obtained, similarly as in (11), according to:

$$
\hat{s}_{k_{0}, n_{0}}=\Re \mathrm{e}\left\{\frac{\theta_{k_{0}, n_{0}}^{*} r_{k_{0}, n_{0}}}{\tilde{C}_{k_{0}}}\right\} .
$$

Thus, in this imperfect CSI case, the ISI and ICI components in the FBMC system before taking the real part will be given by

$$
\begin{aligned}
\frac{\theta_{k_{0}, n_{0}}^{*} r_{k_{0}, n_{0}}}{\tilde{C}_{k_{0}}}= & s_{k_{0}, n_{0}}+\sum_{(k, n) \in \mathcal{R}} \frac{\bar{C}_{k_{0}-k}}{\tilde{C}_{k_{0}}} t_{k, n}\left( \pm s_{k_{0}-k, n_{0}-n}\right) \\
& +\mathrm{j} \sum_{(k, n) \in \mathcal{I}} \frac{\bar{C}_{k_{0}-k}}{\tilde{C}_{k_{0}}} t_{k, n}\left( \pm s_{k_{0}-k, n_{0}-n}\right) .
\end{aligned}
$$

Observe that, due to the phase mismatch between the actual equivalent channel $\bar{C}_{k_{0}-k} \approx \bar{C}_{k_{0}}$ and the estimated one $\tilde{C}_{k_{0}}$ part of the interference in the "imaginary" term in (20) will leak to the real part, which implies that the ISI/ICI components that were cancelled at the detection stage in (9) and (11) when perfect CSI was assumed, now will not disappear, worsening thus the detection quality and increasing the error probability. Graphically, this effect corresponds to the fact that non-shaded elements in Fig. 1 will also be present in the effective ISI/ICI. Observe that the magnitude of the non-shaded elements is much bigger than the magnitude of shaded elements, which were the effective ISI/ICI terms in the perfect CSI case.

The above analytic expression in (20) will be evaluated numerically in the simulations section, in which a comparison between the performance of FBMC and OFDM as a function of the CSI imperfections level will be presented.

\section{ROBUST RECEIVE BEAMFORMER DESIGN}

As expected from the analysis conducted at the end of the previous section, it will be shown, in the simulations section below, that the performance of FBMC degradates much faster than OFDM in imperfect CSI conditions due to the non-cancelled ISI/ICI terms. Therefore, it is of paramount importance to incorporate robust designs in FBMC systems to combat this performance degradation.

In this work, we will consider a robust receive beamvector design based on the transmission of a training sequence. Precisely, the receive beamvector will be designed to minimize the mean square error (MSE) between the processed incoming signal and the training sequence. The complete steps for the robust design are presented in the following:

1) The receiver estimates the channel at each frequency, obtaining $\widetilde{\mathbf{H}}_{k}$, which is a noisy version of the actual channel $\mathbf{H}_{k}$.

2) The transmit beamvector at each frequency $\mathbf{u}_{k}$ is designed as the right singular vector associated with the maximum singular value of the estimated channel $\widetilde{\mathbf{H}}_{k}$ as it has been explained in Section III-B, i.e., $\mathbf{u}_{k}=$ $\sqrt{\tilde{p}_{k}} \tilde{\varphi}_{k}$. For the sake of simplicity, in the imperfect CSI case we will assume that $\tilde{p}_{k}=P_{T} / K, \forall k$, i.e., UPA.

3) The transmitter sends a (known) training real sequence $q_{k, n}$ from $n=0$ to $n=N-1$ so that the receiver can compute the receive beamvectors $\mathbf{v}_{k}$ that minimize the MSE between the training sequence and the filtered received symbols post-beamforming.

The MSE can be easily computed as ${ }^{4}$

$$
\operatorname{mse}\left(\mathbf{v}_{k}\right)=\sum_{n=0}^{N-1}\left(\Re \mathrm{e}\left\{\mathbf{v}_{k}^{H} \mathbf{z}_{k, n}\right\}-q_{k, n}\right)^{2} .
$$

With this definition, the problem of designing the robust receive beamvector can be stated as

$$
\min _{\mathbf{v}_{k}} \operatorname{mse}\left(\mathbf{v}_{k}\right)
$$

Observe that this formulation is different than the one usually encountered in the literature, because, due to the structure of the FBMC transmission scheme, we are only interested in the real part of the detected signal.

\footnotetext{
${ }^{4}$ Observe that, for the expression of the processed signal in the MSE expression in (21) we have used $\Re \mathrm{e}\left\{\mathbf{v}_{k}^{H} \mathbf{z}_{k, n}\right\}$, which is a particular case of the linear complex processing $\mathbf{a}^{H} \mathbf{z}_{k, n}+\mathbf{b}^{H} \mathbf{z}_{k, n}^{*}$. In Appendix A we prove that the choice $\mathbf{b}^{H}=\mathbf{a}^{T}$ is indeed optimal and leads to $\mathbf{a}^{H} \mathbf{z}_{k, n}+\mathbf{a}^{T} \mathbf{z}_{k, n}^{*} \propto \Re \mathrm{e}\left\{\mathbf{a}^{H} \mathbf{z}_{k, n}\right\}$.
} 
We now proceed to the computation of the optimal robust receive beamvector. Defining

$$
\begin{aligned}
\mathbf{R}_{k} & =\sum_{n=0}^{N-1} \mathbf{z}_{k, n} \mathbf{z}_{k, n}^{H} \\
\overline{\mathbf{R}}_{k} & =\sum_{n=0}^{N-1} \mathbf{z}_{k, n} \mathbf{z}_{k, n}^{T} \\
\mathbf{p}_{k} & =\sum_{n=0}^{N-1} \mathbf{z}_{k, n} q_{k, n} \\
Q_{k} & =\sum_{n=0}^{N-1} q_{k, n}^{2}
\end{aligned}
$$

the MSE can be expressed, after a few cumbersome operations, as

$$
\begin{aligned}
\operatorname{mse}\left(\mathbf{v}_{k}\right)= & \frac{1}{2} \mathbf{v}_{k}^{H} \mathbf{R}_{k} \mathbf{v}_{k}-\mathbf{v}_{k}^{H} \mathbf{p}_{k}-\mathbf{v}_{k}^{T} \mathbf{p}_{k}^{*} \\
& +\frac{1}{4} \mathbf{v}_{k}^{H} \overline{\mathbf{R}}_{k} \mathbf{v}_{k}^{*}+\frac{1}{4} \mathbf{v}_{k}^{T} \overline{\mathbf{R}}_{k}^{*} \mathbf{v}_{k}+Q_{k}
\end{aligned}
$$

where, in the derivations, we have used

$$
\Re \mathrm{e}\left\{\mathbf{v}_{k}^{H} \mathbf{z}_{k, n}\right\}=\frac{1}{2}\left(\mathbf{v}_{k}^{H} \mathbf{z}_{k, n}+\mathbf{z}_{k, n}^{H} \mathbf{v}_{k}\right) .
$$

Now, it is not difficult to compute the gradient of the MSE with respect to the receive beamvector $\mathbf{v}_{k}$ as

$$
\nabla_{\mathbf{v}_{k}^{H} \text { mse }}=\frac{1}{2} \mathbf{R}_{k} \mathbf{v}_{k}+\frac{1}{2} \overline{\mathbf{R}}_{k} \mathbf{v}_{k}^{*}-\mathbf{p}_{k} .
$$

Equating last expression to zero we obtain the optimality condition for the receive beamvector at each frequency:

$$
\mathbf{R}_{k} \mathbf{v}_{k}+\overline{\mathbf{R}}_{k} \mathbf{v}_{k}^{*}=2 \mathbf{p}_{k},
$$

where we have used the slight abuse of notation to designate with the same symbol, $\mathbf{v}_{k}$, the variable and the optimal value.

From (31), a closed form expression for the optimal beamvector $\mathbf{v}_{k}$ can be computed as indicated in the following. Let's denote the real and imaginary parts of a complex vector/matrix by the superscripts $(\cdot)^{(r)}$ and $(\cdot)^{(i)}$, respectively. Now, we can rewrite (31) in real-valued matrix form according to

$$
\begin{aligned}
& \left(\begin{array}{c}
\mathbf{v}_{k}^{(r)} \\
\mathbf{v}_{k}^{(i)}
\end{array}\right) \\
& =2\left(\begin{array}{cc}
\mathbf{R}_{k}^{(r)}+\overline{\mathbf{R}}_{k}^{(r)} & \overline{\mathbf{R}}_{k}^{(i)}-\mathbf{R}_{k}^{(i)} \\
\mathbf{R}_{k}^{(i)}+\overline{\mathbf{R}}_{k}^{(i)} & \mathbf{R}_{k}^{(r)}-\overline{\mathbf{R}}_{k}^{(r)}
\end{array}\right)^{-1}\left(\begin{array}{c}
\mathbf{p}_{k}^{(r)} \\
\mathbf{p}_{k}^{(i)}
\end{array}\right) .
\end{aligned}
$$

The performance of the proposed robust beamforming scheme will be studied in the simulations section.

\section{Simulations}

For the sake of simplicity, in this section we will consider a very basic model for the channel estimation error. Precisely, we will assume that $\widetilde{\mathbf{H}}_{k}=\mathbf{H}_{k}+\boldsymbol{\Delta}_{k}$ where the entries of $\boldsymbol{\Delta}_{k}$ are independent zero-mean circularly symmetric complex Gaussian random variables with power equal to $P_{E}$. This

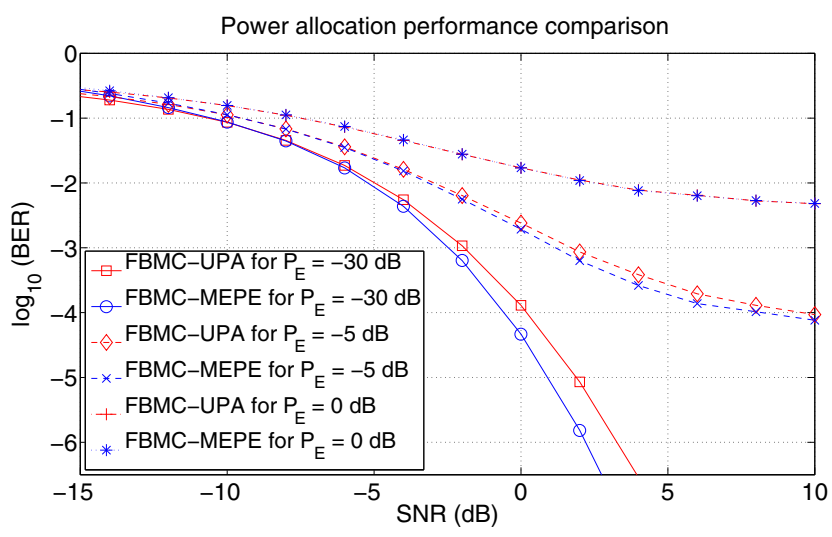

Fig. 4. Performance comparison for different power allocation strategies.

model would fit, e.g., in a situation where the receiver imperfectly estimates the channel in the frequency domain and then feeds back this estimation to the transmitter through an ideal feedback link. It must be highlighted that the results obtained in this section also hold for other models for the channel estimation error. However, the simple dependence of our model on exclusively the parameter $P_{E}$ makes it suitable for the purposes of this paper.

Similarly as in Section II-B, for the FBMC system we have taken the design in [9] with filter length $L=2048$, symbol period $\tau=256$, and inverse of the frequency separation $M=512$. For the OFDM system, the $\mathrm{CP}$ has been assumed to be one fourth of the total symbol duration. The PAM modulation for FBMC is BPSK and QPSK has been considered in OFDM so that the rate is the same in both systems, the total transmitted power has been normalized to $P_{T}=K$, and the SNR of the system is given by $P_{T} / \sigma^{2}$, with $\sigma^{2}$ being the power of the noise vector $\mathbf{w}(m)$ defined in Section III-A. The channel has been chosen to be a Rayleigh distributed MIMO channel, whose length has been fixed to 10 with an exponentially decaying power delay profile (the delay spread is equal to 5 times the sampling period). We have considered different configurations for the numbers of transmit and receive antennas.

First of all, we have studied the effects in the BER of the different power allocation strategies for non-robust FBMC systems. In Fig. 4, it can be readily seen that the MEPE power allocation yields always better BER results than UPA. However, as the estimation error $P_{E}$ increases the advantage margin becomes narrower.

Next, we have compared the performance of the non-robust FBMC and OFDM systems for different qualities of the CSI. In Fig. 5 we have depicted the BER performance of the FBMC as a function of the SNR for two different values of $P_{E}$, which models the degree of imperfection in the CSI. As expected, for low values of $P_{E}$ the FBMC system yields a lower BER because OFDM wastes some power transmitting the CP. For higher values of $P_{E}$, OFDM performs better because FBMC is suffering from ISI and ICI due to the estimation errors.

Next, we introduce the robust FBMC system, as described 


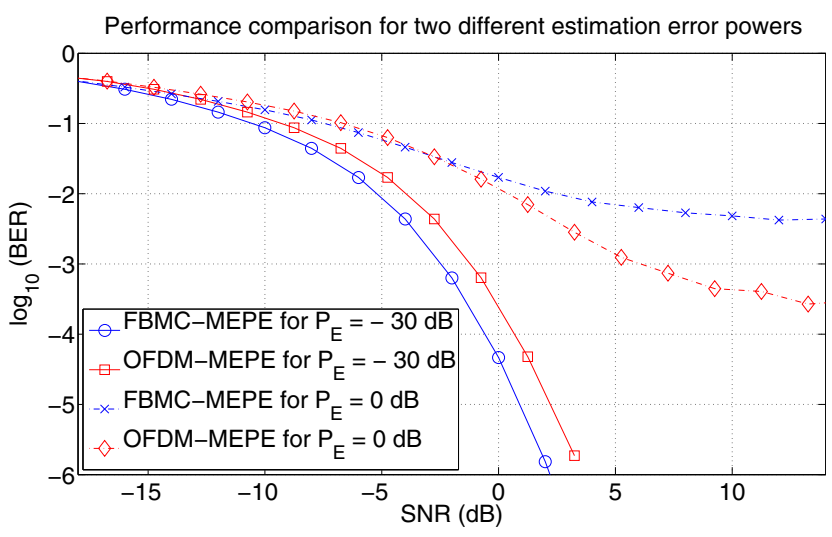

Fig. 5. Performance comparison for different estimation error powers.

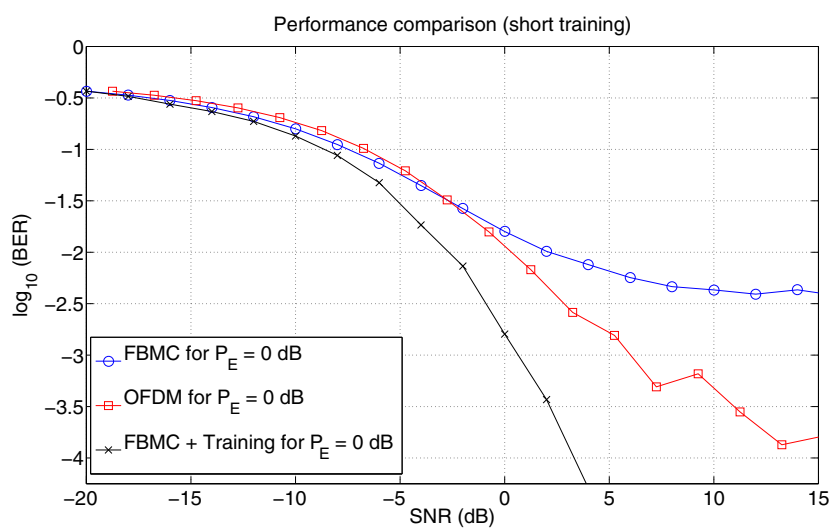

Fig. 6. Performance comparison of non-robust FBMC, OFDM, and robust FBMC for a short training sequence and imperfect CSI.

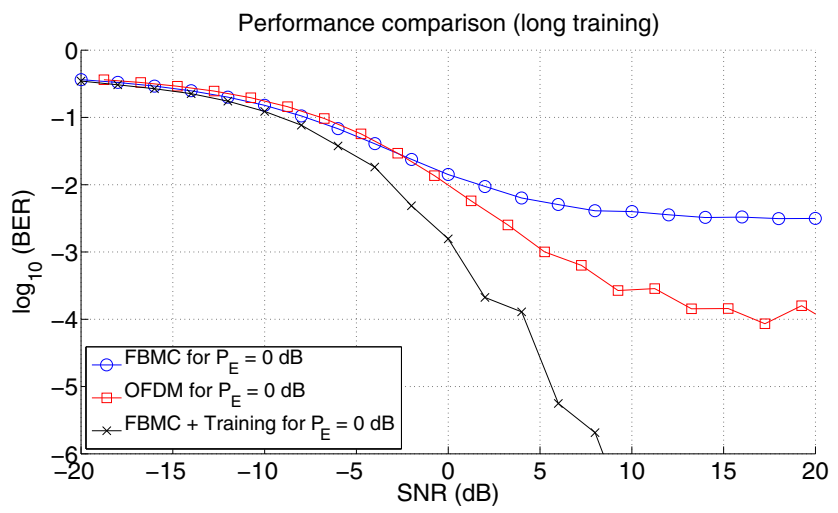

Fig. 7. Performance comparison of non-robust FBMC, OFDM, and robust FBMC for a long training sequence and imperfect CSI.

in Section IV. We have considered two lengths for the training sequence (short, $\mathrm{N}=128$, and long, $\mathrm{N}=1024$ ) and also we have considered two different qualities for the CSI (almost perfect CSI, $P_{E}=-30 d B$, and imperfect CSI, $P_{E}=0 d B$ ). The BER performance in all these scenarios is shown in Figs. 6, 7, 8, and 9 .

As it can be seen from Fig.6, in the almost perfect CSI case and short training sequence, robust FBMC performance is very close to that of non-robust FBMC but it is slightly worse. This

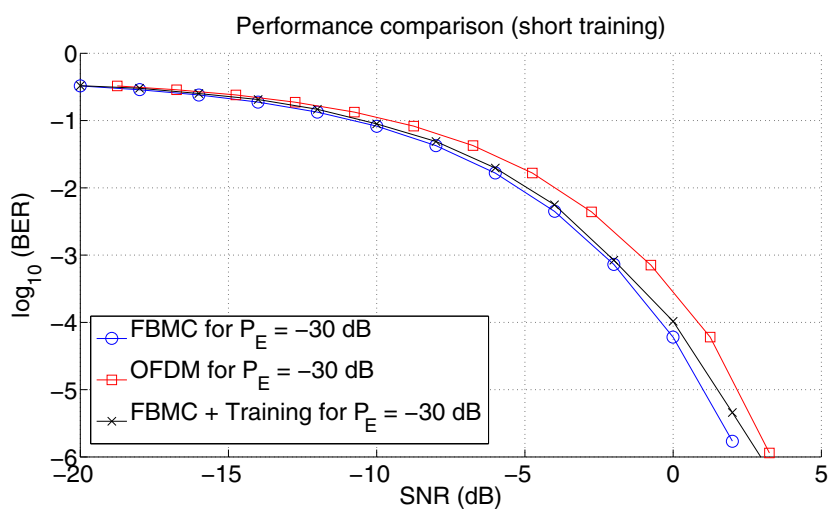

Fig. 8. Performance comparison of non-robust FBMC, OFDM, and robust FBMC for a short training sequence and almost perfect CSI.

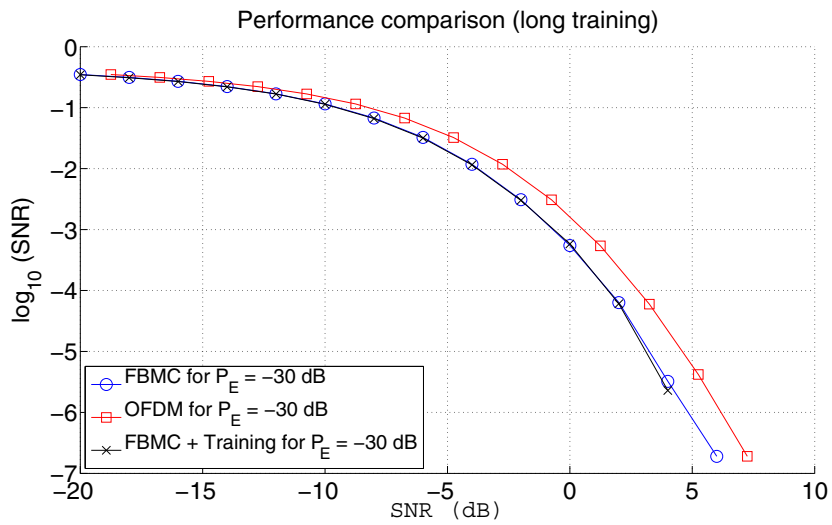

Fig. 9. Performance comparison of non-robust FBMC, OFDM, and robust FBMC for a long training sequence and almost perfect CSI.

is due to the fact that the short training sequence does not carry enough information for the receiver to design a better receive beamvector than the left singular vector $\boldsymbol{\psi}_{k}$, which is used in the non-robust FBMC and it is optimal in perfect CSI scenarios. In Fig. 7, a longer training sequence is considered and the robust FBMC performance matches that of the nonrobust one. It is worth to highlight that the performance of the robust FBMC is better than the performance of OFDM.

In Figs. 8 and 9, we have considered a scenario with imperfect CSI, $P_{E}=0 d B$. In this case, it can be seen that, for both training sequence lengths, the performance of robust FBMC clearly outperforms that of OFDM and non-robust FBMC, validating, thus, our proposed robust scheme.

Finally, in Fig. 10 we have chosen a working SNR of 0 $\mathrm{dB}$ and have plotted the BER as a function of the estimation error power $P_{E}$ for the three schemes under consideration. In this case, it can be clearly seen that there is a crossing point between the non-robust FBMC and OFDM BER curves, which further corroborates the fact that FBMC degrades faster than OFDM as the estimation error power increases. Moreover, it can be seen that the performance of robust FBMC is neatly superior to that of non-robust FBMC and OFDM for the whole range of CSI imperfections level. 


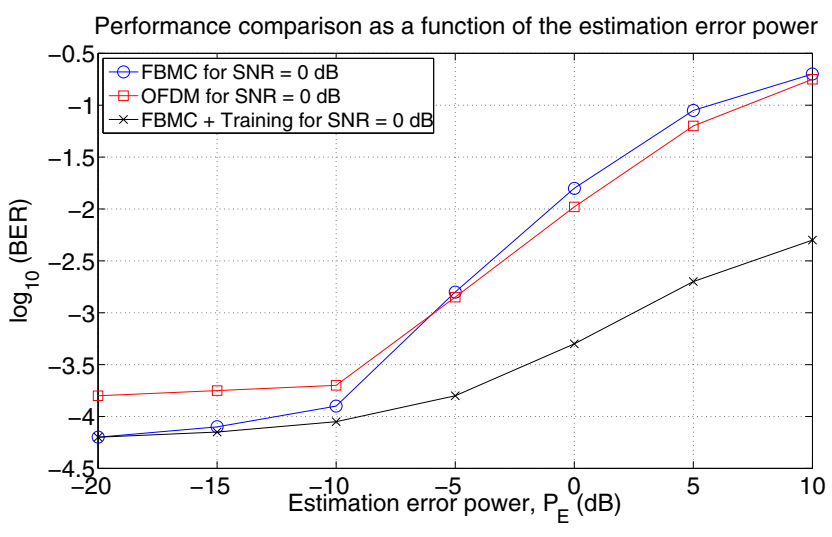

Fig. 10. Performance comparison as a function of the channel estimation error power, $P_{E}$

\section{CONCLUSION}

In this work, we have studied the perforance of FBMC systems in scenarios with multiple antennas under different quality degrees of the CSI. We have shown that the performance degradation of non-robust FBMC is worse than the degradation suffered in OFDM systems due to the fact that, with imperfect CSI, additional significant ISI/ICI terms appear in FBMC and not in OFDM. To cope with this problematic situation, we have derived a novel robust receive beamforming for FBMC systems and have shown its superior BER performance when compared with OFDM.

\section{APPENDIX A}

The aim of this appendix is to prove the optimality of the robust receive beamvector proposed in Section IV. We define the MSE for the case of computing a general linear combination of the real and imaginary parts of received vector z. To this end we use two different beamvectors, a and $\mathbf{b}$, which are applied to the received vector $\mathbf{z}$ and to its conjugate $\mathbf{z}^{*}$, respectively. For the sake of simplicity we omit the frequency carrier subindex $k$ and express the MSE as

$$
\operatorname{mse}(\mathbf{a}, \mathbf{b})=\sum_{n=0}^{N-1}\left|\mathbf{a}^{H} \mathbf{z}_{n}+\mathbf{b}^{H} \mathbf{z}_{n}^{*}-q_{k, n}\right|^{2} .
$$

With this definition, the problem of designing the robust receive beamvectors can be stated as

$$
\min _{\mathbf{a}, \mathbf{b}} \operatorname{mse}(\mathbf{a}, \mathbf{b})
$$

From (23)-(26) the MSE can be expressed as

$$
\begin{aligned}
\operatorname{mse}(\mathbf{a}, \mathbf{b})= & \mathbf{a}^{H} \mathbf{R} \mathbf{a}+\mathbf{a}^{H} \overline{\mathbf{R}} \mathbf{b}-\mathbf{a}^{H} \mathbf{p} \\
& +\mathbf{b}^{H} \overline{\mathbf{R}}^{*} \mathbf{a}+\mathbf{b}^{H} \mathbf{R}^{*} \mathbf{b}-\mathbf{b}^{H} \mathbf{p}^{*} \\
& -\mathbf{p}^{H} \mathbf{a}-\mathbf{p}^{H} \mathbf{b}+Q
\end{aligned}
$$

Computing the gradient of the MSE with respect to the receive beamvectors $\mathbf{a}$ and $\mathbf{b}$ as

$$
\begin{aligned}
\nabla_{\mathbf{a}^{H} \mathrm{mse}} & =\mathbf{R} \mathbf{a}+\overline{\mathbf{R}} \mathbf{b}-\mathbf{p} \\
\nabla_{\mathbf{b}^{H}} \mathrm{mse} & =\overline{\mathbf{R}}^{*} \mathbf{a}+\mathbf{R}^{*} \mathbf{b}-\mathbf{p}^{*}
\end{aligned}
$$

and equating both expressions to zero, we obtain the optimality conditions for the receive beamvectors. Then, it is easy to see that choosing $\mathbf{b}=\mathbf{a}^{*}$ in (38) and conjugating the whole expression we obtain the same condition as in (39), which implies that the choice $\mathbf{b}=\mathbf{a}^{*}$ (i.e., $\mathbf{b}^{H}=\mathbf{a}^{T}$ ) is optimal.

\section{REFERENCES}

[1] P. P. Vaidyanathan, "Multirate digital filters, filter banks, polyphase networks, and applications: A tutorial," Proceedings of the IEEE, vol. 78, no. 1, pp. 56-93, 1990.

[2] G. Li and G. Stüber, Orthogonal frequency division multiplexing for wireless communications. Springer, 2006.

[3] P. Siohan, C. Siclet, and N. Lacaille, "Analysis and design of OFDM/OQAM systems based on filterbank theory," IEEE Trans. on Signal Processing, vol. 50, no. 5, pp. 1170-1183, 2002.

[4] H. Bölcskei, Advances in Gabor Analysis. Birkhäuser, 2003, ch. Orthogonal frequency division multiplexing based on offset QAM, pp. 321-352. [Online]. Available: http://www.nari.ee.ethz.ch/commth/pubs/p/gabor_book_chap

[5] G. J. Foschini and M. J. Gans, "On limits of wireless communications in a fading environment when using multiple antennas," Wireless Personal Communications, vol. 6, no. 3, pp. 311-335, 1998.

[6] A. Pascual-Iserte, A. I. Pérez-Neira, and M. A. Lagunas, "On power allocation strategies for maximum signal to noise and interference ratio in an OFDM-MIMO system," IEEE Trans. on Wireless Communications, vol. 3, no. 3, pp. 808-820, May 2004.

[7] M. Bengtsson and B. Ottersten, "Optimal and suboptimal transmit beamforming," Handbook of Antennas in Wireless Communications, 2001.

[8] C. Lélé, J.-P. Javaudin, R. Legouable, A. Skrzypczak, and P. Siohan, "Channel estimation methods for preamble-based OFDM/OQAM modulations," European Transactions on Telecommunications, vol. 19, no. 7, pp. 741-750, 2008.

[9] M. G. Bellanger, "Specification and design of a prototype filter for filter bank based multicarrier transmission," in IEEE International Conference on Acoustics Speech and Signal Processing, (ICASSP'01), 2001.

[10] ICT-PHYDYAS, "D3.1 - Equalization and demodulation in the receiver (single antenna)," deliverable available at http://www.ict-phydyas.org, Tech. Rep., July 2008. 